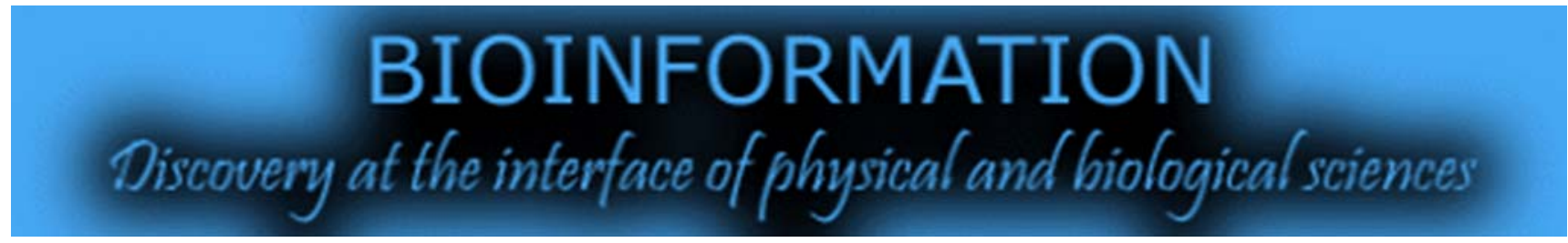

\title{
Selection of herbal therapeutics against deltatoxin mediated Clostridial infections
}

\author{
Sinosh Skariyachan $^{1 *}$, Arpitha Badarinath Mahajanakatti ${ }^{1}$, Narasimha Sharma ${ }^{1}$, \\ Murugan Sevanan'
}

${ }^{1} \mathrm{R} \&$ D Center, Department of Biotechnology, Dayananda Sagar College of Engineering, Bangalore-560 078, Karnataka, India; ${ }^{2}$ Department of Biotechnology, Karunya University, Coimbatore- 641 114, Tamilnadu, India; Sinosh Skariyachan - Email: sinoshskariya@gmail.com; Phone: +91 9739654015, +91 8042161748 (Off); Fax: 080-42161747; *Corresponding author

Received July 25, 2011; Accepted July 29, 2011; Published August 02, 2011

\begin{abstract}
:
Clostridium perfringens (a versatile pathogenic bacterium) secretes enterotoxins (the deltatoxin, virulent factor) and causes food borne gastroenteritis and gasgangrene. The organism was isolated and characterized from improperly cooked meat and poultry samples. The isolated organism showed multiple drug resistance indicating that the treatment is challenging. Hence, there is need for improved therapeutic agents. The rational design of improved therapeutics requires the crystal structure for the toxin. However, the structure for the toxin is not yet available in its native form. Thus, we modeled the toxin structure using $\alpha$ hemolysin of Staphylococcus aureus (PDB: 3M4D chain A) as template. The docking of the toxin with the herbal extract curcumin (1,7-bis(4-hydroxy-3methoxyphenyl)hepta-1,6-diene-3,5-dione) showed a binding energy of - $8.6 \mathrm{Kcal} / \mathrm{mol}$, in comparison to the known antibiotic Linezolid with binding energy of -6.1 $\mathrm{Kcal} / \mathrm{mol}$. This data finds application in the design and development of novel compounds against the deltatoxin from Clostridium perfringens.
\end{abstract}

\section{Background:}

Clostridium perfringens is a gram-positive, anaerobic, spore forming and pathogenic bacterium. The bacterium belongs to major intestinal flora of human and animal. The pathogenesis of the organism includes gas gangrene, necrotizing fasciitis, diarrhea brain abscess in pigs, calves, chickens, and other animals. The rapid generation time and heat resistance ability makes the organism a major food-borne pathogen [1]. The outbreak of Clostridium perfringens is regarded as the second most bacterial food poisoning in USA and UK, where cases occurring annually are 250,000 and 85,000 respectively [2]. Economic losses due to medical care and productivity loss from single food poisoning amount to several hundred million dollars per year [3]. The major virulent factors involved in gastroenteritis and other infections are extracellular toxins produced by the bacteria. C. perfringens is classified into five serotypes (A, B, C, D and E) based on the type of enterotoxins. Type A and $\mathrm{C}$ strains are the most dangerous pathogens as it is implicated in human diseases. Type A strains cause the most destructive disease called gas gangrene which is characterized by rapid destruction of tissue with the production of gas. Type B, C, D and E are mainly responsible for veterinary infections [4] Deltatoxin is one of the five hemolysins released by most of the Clostridium perfringens which plays most important role in the gasgangrene and gastroenteritis. The organism is developing resistance to most conventional classes of known antibiotics and has emerged as "superbugs". So there is an emergency to address the problem by finding better therapeutic substances which could replace the antibiotics. The active substances present in many medicinal plants could be used as therapeutic alternatives against Clostridial infections [5]. The native structure of the deltatoxin was not reported in the structural databases. Homology modeling is a computer aided approach to generate all possible folds and conserved motifs responsible for the actual function of proteins. It has proven to be the method of choice to generate a reliable $3 \mathrm{D}$ model of a protein from its amino acid sequence (target) by identifying a homologous protein with a known structure (template). The comparative modeling of hypothetical protein consists of target selection, template identification, fold assignment, structural alignment, model building and model evaluation [6]. Prediction of receptor-ligand interaction is the fundamental concept of drug designing. Structure prediction enables to explain the mechanisms of interaction between G-protein coupled receptors and variety of ligands, enzymes, ion channels and current drugs. 3D model prediction and target identification have profound scope in the field of new generation drug development process [7]. The prediction of putative protein-ligand binding conformations by computational docking has pronounced impact for discovering new generation lead molecules.

\section{Methodology:}

Microbial characterization and study of multidrug resistance:

Clostridium perfringens is a hyperthermophilic bacteria and it survives in any kind of food items cooked improperly. A total of 32 fried samples of meat (10 samples), chicken and poultry (12 samples each) were collected from different regions of Coimbatore, India. The presumptive detection of Closrtidium perfringens was carried out by pour plate method on selective Tryptose Sulphate Cycloserine (TSC) agar. The confirmed and completed tests were performed by standard microbiological and biochemical tests. There are reports that many bacteria developed multiple drug resistance towards conventionally used antibiotics and emerged as "superbugs". The antibiotics sensitivity testing with the isolated organisms is a critical step to understand the drug resistance 
and new drug discovery mechanisms. Hence, the isolated organism is tested for antibiotic sensitivity patterns by Kirby- Bauer disc diffusion method.

\section{Computer aided drug discovery:}

Since the isolated organism showed resistance to many antibiotics, the drug of choice against the infection became limited. Thus, a novel approach has to be developed to design new therapeutic substances; one such method is called computer aided screening. Deltatoxin is the major virulent factor and probable drug target. So the structural studies and fold recognition of deltatoxin is critical step to develop new lead molecules. But the crystal structure of deltatoxin is not present in its native form. The 3D structure of toxin could be modeled by comparative modeling using its amino acid sequence.

\section{Sequence retrieval and template selection:}

The amino acid sequence of deltatoxin was retrieved from GenBank (GI 194719328) [8]. Since the quality of the model depends on the availability of good template, it is important to identify the best template structure. The best homologous protein was selected by PSI- BLAST [9] based on the percentage of identity and similarity. A multiple sequence analysis has been performed by T-COFFEE [10] to analyze the evolutionary conservation among the sequences. The phylogenetic characterization was carried out using NJ PLOT [11]. All these steps are essential factors for the selection of best template.

\section{Model building and validation:}

The crystal structure of drug target (Deltatoxin) is not available in its native form. Thus, the protein was modeled using MODELLER 9v9. It is based on satisfaction of spatial restraints derived from the alignment and Probability Density Functions (PDFs) [7]. The X-ray crystal structure of $\alpha$ - hemolysin [12] of Staphylococcus aureus (PDB ID: 3M4D, Chain A) was identified as the bes template. The initial model building and structural alignment was performed and the modeled protein was visualized using UCSF CHIMERA [13]. Energy minimization of the generated model was done through CHARMM [14] Quasi-Newton Mechanics [15] and GBSA Surface Potential [16]. Parameters like covalent bond distances and angles; stereo-chemical validation and atom nomenclature were validated using PROCHECK [17] and overall quality factor of non-bonded interactions between different atoms types were calculated by ERRAT program [18]. DaliLite [19] is used to calculate Root Mean Square Deviation (RMSD) between the set of targets and template protein to see how much modeled protein deviates from the template protein structure. The hypothetical model was then deposited to Protein Model Data Base.

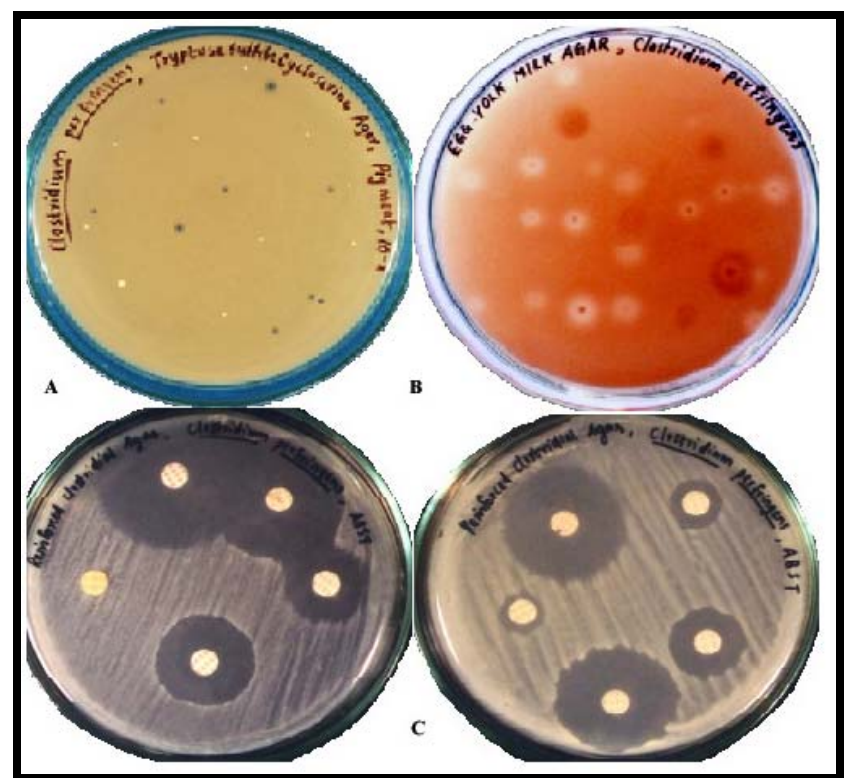

Figure 1: Clostridium perfringens, a notorious pathogenic bacterium, isolated from improperly cooked food samples. (A) The growth is indicated by the formation of black colored colonies on selective medium. (B) The enterotoxin production of the organism characterized by lecithinase activity, an opaqueness and halo around the colonies in the egg-yolk milk agar. (C) Multidrug resistance patterns of the isolated organism towards Amphotericin B (20 $\mathrm{mcg} / \mathrm{disc})$, Polymyxin B $(50 \mathrm{mcg} / \mathrm{disc})$ and Streptomycin $(25 \mathrm{mcg} / \mathrm{disc})$. The organism is moderately sensitive to Vancomycin $(15 \mathrm{mcg} /$ disc $)$, Erythromycin
$(30 \mathrm{mcg} / \mathrm{disc})$ and Bacitracin $(10 \mathrm{mcg} / \mathrm{disc})$ indicating that antibiotics are not suitable drug of choice against Clostridial infection.

\section{Selection of ligands and docking studies:}

Since the organism has developed resistance towards many conventionally used antibiotics, it is essential to screen for better therapeutic substances. It has been known that many herbal based compounds have high druggish activity and binding affinity towards deltatoxin. Selection and screening of molecules with good pharmacophoric and druggish activity is the critical step in computer aided drug screening. Based on extensive literature studies, 70 plant extracts and 5 antibiotics were identified. The drug likenesses and pharmacophoric features of ligands were studied by Lipinski's rule of 5. Protein ligand docking was performed using AUTODOCK 4.2 [20] by Lamarckian genetic algorithm. The catalytic and binding site of the target has been identified by auto grid. The structure and chemical properties of the active site allow the recognition and binding of the ligand. Around 2,500,000 bioactive conformations were generated by 10 iterations and the best conformations were screened in terms of lowest binding energy generated in the clustering histogram.

\section{Discussion:}

The improper cooking of food items, especially meat and meat products, results in the survival of hyperthermophilic Clostridia which may cause gastroenteritis and other health hazards. We have isolated and characterized Clostridium perfringens from fried samples of meat products (Table 1 See supplementary material). We have noticed that the food samples were consumed by lots of people without any hygienic practices that may result in sudden outbreak of food poisoning. The isolated organism from the collected samples is illustrated in Figure 1A; the organism produces black colored colonies on the selective TSC medium. The virulent factors of the organism are enterotoxins, mainly delta, which is characterized by lecithinase activity, a zone of inhibition around the colonies due to toxin production, is illustrated in Figure 1B, The major problem pointed in this study is the necessity to develop other therapeutic substances, because the isolated organism is resistant to many antibiotics and treatment with conventional drug becomes challenging in future. We have tested the antibiotic sensitivity patterns of isolated bacteria, illustrated in Figure 1C. The antibiogram clearly showed that the organism is resistant to Streptomycin, Polymyxin-B and Amphotericin-B and moderately sensitive to Bacitracin, Erythromycin and Vancomycin which are all currently used drugs against the infection

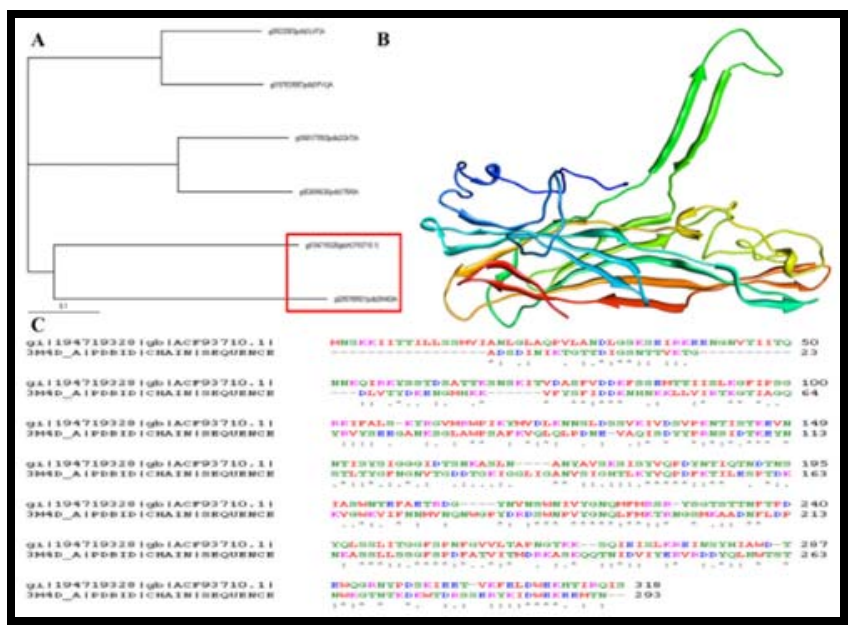

Figure 2: Generation of hypothetical model of deltatoxin and selection of best template for modeling ( $\mathrm{A}$ and $\mathrm{C}$ ). The phylogram and alignments are indicating that the sequences of deltatoxin shared evolutionary relatedness to $\alpha$-hemolysin (A chain) of Staphylococcus areus (marked in red box). (B) The generated 3D model of protein consists of stable secondary structure (helices and sheets) which gives the catalytic sites for drug interaction.

Computer aided screening is an ideal platform to develop novel compounds against many diseases. As mentioned deltatoxin is the major virulent factor for the infections caused by Clostridium perfringens. The 3D structure of deltatoxin is not available but it is very essential for rational drug discovery. We have identified all the possible folds and generated a 3D model of deltatoxin from its protein sequence (GenBank, GI: 194719328) by comparative modeling. The protein has 318 amino acids and encodes transposase genes (957 bps) which act as major functional element of the toxin. 
The best template for homology modeling, selected based on the similarity search and phylogenetic characterization is shown in Figure 2A \& 2C. Out of six homologous sequences, the crystal structure of M113N mutant of $\alpha$ hemolysin of Staphylococcus aureus (PDBID: 3M4D, chain A) was identified as best template with $33 \%$ identity and 53\% similarity. The resolution of template structure was $1.9 \mathrm{~A}^{\circ}$ and $\mathrm{R}$ value was 0.24 . The molecular weight is $35,520 \mathrm{kDa}$ and it consists of 293 amino acids. The secondary structure prediction revealed that $56.29 \%$ of random coil, $31.13 \%$ of extended strands, $7.86 \%$ of alpha helices and $4.72 \%$ of beta turns. The toxin consists of hydrophobic transmembrane helix between the amino acids 40 and 57 . The generated 3D model is illustrated in Figure 2B. The model has same structural conformation as the template which is very essential for receptor - ligand interaction. The 3D model is refined using energy minimization by molecular dynamic methods such as CHARMM, Quasi-Newton Mechanics and GBSA surface potential, given the stable conformation of the model. The Ramachandran plot (generated by PROCHECK) validated the quality of homology model, $87.9 \%$ residues are in most favored region implies the quality of the model is good. The overall quality factor of non-bonded interactions between different atoms identified by ERRAT was $58.3 \%$. The backbone RMSD estimated from superimposition of the template and target was found to be $1.2 \AA$ also reveals the quality of the model is good. The model was deposited to Protein Model Database, a repository of storing manually built 3D models of proteins, and it can be downloaded by PM0076541.

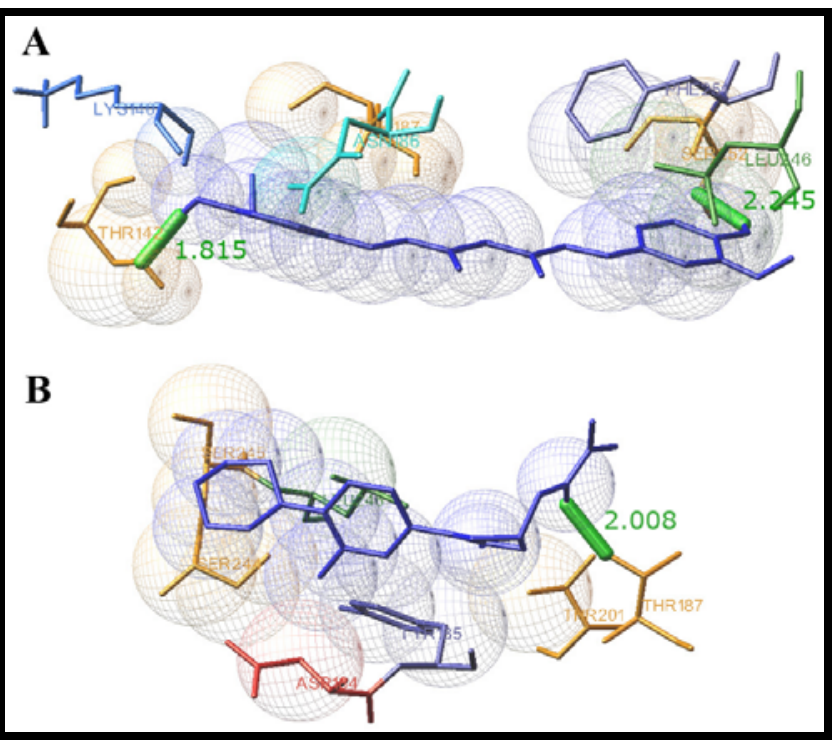

Figure 3: Docked structures of Curcumin (A) and Linezoid (B) with deltatoxin. Turmeric compound Curcumin showed better binding affinity to deltatoxin than Linezoid explains the therapeuctic value of plant compounds over antibiotics. The ligand - receptor interaction in the case of Curcumin is stabilized by two $\mathrm{H}$ bonds (represented as green colored stick, length-1.815 $\mathrm{A}^{\circ}$ and $2.245 \mathrm{~A}^{\circ}$ ) and the amino acid residues interacting are LYS140, THR142, THR148, ASN186, THR 187, LEU246 and SER252 making the interaction more stronger and stable (A). The binding energy of docked complex was found to be $-8.6 \mathrm{kcal} / \mathrm{mol}$ implies more stable docking. The interaction of Linezoid and deltatoxin is stabilized by only one $\mathrm{H}$ bond (green colored stick of $2.0 \mathrm{~A}^{\circ}$ lengths) and the interacting residues are ASP184, THR185, THR187, THR201, SER 244, SER 245 and LEU246 indicating that the interaction less stronger than that of Curcumin. The binding energy of the docked complex was found to be $-6.08 \mathrm{kcal} / \mathrm{mol}$, which indicates that the docked complex is less stable compared to curcumin.

Docking simulations are the best method to study receptor - ligand interactions in drug designing. The docking was performed to compare the binding efficiency of antibiotics and herbal compounds towards deltatoxin. There are reports on the antimicrobial potentials of many plant compounds against Clostridial infection. In this perspective, 70 herbal extracts were identified and screened by Lipinski Rule of 5, implies that molecules should contain less than 10 Hbond acceptors and 5 Hbond donors. The calculated $\log \mathrm{P}$ value should be less than 5 and the molecular weight should be less than $500 \mathrm{~g} / \mathrm{mol}$. All compounds satisfied the rule and the molecules were subjected to docking studies (Table 2 See supplementary material). Out of 70 molecules tested, Curcumin, Eugenol, Palmatine, Eucalyptol and Chrysin showed best interactions with deltatoxin whose binding energies are $-8.60 \mathrm{kcal} / \mathrm{mol},-6.18$ $\mathrm{kcal} / \mathrm{mol},-5.72 \mathrm{kcal} / \mathrm{mol},-5.69 \mathrm{kcal} / \mathrm{mol}$ and $5.42 \mathrm{kcal} / \mathrm{mol}$ respectively. Curcumin, a curcuminoid, isolated from Indian spice turmeric (Curcuma longa) was the best inhibitor than the antibiotic Linezoid. The docked complex is stabilized by two hydrogen bonds and the interacting amino acids are LYS140, THR142, THR148, ASN186, THR 187, LEU246 and SER252 which are illustrated in Figure 3A. The interactions were strong because of more number of interacting residues, number of hydrogen bonds and shorter bond length. The antibiotics Linezoid, Clindamycin, Penicillin, Chloramphenicol and Metronidazole were docked with deltatoxin. Linezoid and Clindamycin showed best interactions to the target protein with binding energies of $-6.08 \mathrm{kcal} / \mathrm{mol}$ and $-5.69 \mathrm{kcal} / \mathrm{mol}$ respectively. The interacting residues between Linezolid and deltatoxin were ASP184, THR185, THR187, THR201, SER 244, SER 245, LEU246 and it is stabilized by one hydrogen bond illustrated in Figure 3B. The interactions with the antibiotics were not stable enough to produce good docked conformations compared to plant derived molecules. The docking studies clearly explains Curcumin is interacting more efficiently with deltatoxin than antibiotic Linezolid and it could be a new lead molecule against the deltatoxin mediated clostridial infection. Similarly the other plant molecules tested in the study can also be used as therapeutic alternatives because it is more effectively interacting with deltatoxin than antibiotics.

\section{Conclusion:}

The study concluded that computer aided drug discovery is an emerging and effective alternative for identification of novel therapeutic substances. Several naturally available herbal compounds are identified and their effectiveness against Clostridial infection is tested by molecular docking. Curcumin, Eugenol and similar kinds of herbal based compounds were identified to be effective inhibitors against deltatoxin. The binding energies of herbal based compounds are less than that of antibiotics hence; herbal medicines could solve all problems of multiple drug resistance by many bacteria. The study also helpful for pharmaceutical sectors as computer aided screening would reduce the complexities involved in the discovery and development of new lead molecules.

\section{Acknowledgements:}

The authors thankfully acknowledge the R \& D Centre of Life Sciences and Engg, Dayananda Sagar Institutions for providing the necessary facilities and also grateful to Dr. P.S Rao, Director Life Sciences and Engg. for his constant support and encouragement throughout the study.

\section{References:}

[1] Miyamoto K et al. PLOS One. 2011 6: e20376 [PMID: 21655254]

[2] Lindström M et al. Food Microbiol. 2011 28: 192 [PMID: 21315973]

[3] Li J \& McClane BA. PLoS Pathog. 2008 4: e1000056 [PMID: 18451983]

[4] Sengupta N et al. Infect Immun. 2010 78: 3957 [PMID: 20605988]

[5] Woodford N \& Livermore DM. J Infect. 200959 Suppl 1: S4 [PMID: 19766888]

[6] Kelm S et al. Bioinformatics 2010 26: 2833 [PMID: 20926421]

[7] Hopkins AL \& Groom CR. Nat Rev Drug Discov. 2002 1: 727 [PMID: 12209152]

[8] Manich M et al. PLOS One. 2008 3: e3764 [PMID: 19018299]

[9] Johnson M et al. Nucleic Acids Res. 2008 36: W5 [PMID: 18440982]

[10] Notredame CJ et al. J Mol Biol. 2000 302: 205 [PMID: 10964570]

[11] Perrière G \& Gouy M. Biochimie. 1996 78: 364 [PMID: 8905155]

[12] Banerjee A et al. Proc Natl Acad Sci U S A. 2010 107: 8165 [PMID 20400691]

[13] Pettersen EF et al. J Comput Chem. 2004 25: 1605 [PMID: 15264254]

[14] Brooks BR et al. J Comput Chem. 2009 30: 1545 [PMID: 19444816]

[15] Ulrich P et al. Proteins. 1997 27: 367 [PMID: 9094739]

[16] Onufriev A et al. J Comput Chem. 2002 23: 1297 [PMID: 12214312]

[17] Laskowski RA. J Appl Cryst. 1993 26: 283

[18] Colovos C \& Yeates TO. Protein Sci. 1993 9: 1511 [PMID: 8401235]

[19] Holm L \& Park J. Bioinformatics 2000 16: 566 [PMID: 10980157]

[20] Morris GM et al. J Comput Aided Mol Des. 1996 10: 293 [PMID: 8877701]

Edited by P Kangueane

Citation: Skariyachan et al. Bioinformation 6(10): 375-379 (2011)

License statement: This is an open-access article, which permits unrestricted use, distribution, and reproduction in any medium, for non-commercial purposes, provided the original author and source are credited. 


\section{Supplementary material:}

Table 1: The biochemical characterization of isolated organism. Black colored colonies on TSC plate, $\beta$-hemolytic colony characterized by double zone of inhibition, growth at strict anaerobic condition, stormy fermentation and lecithinase activity are confirmed that the isolated organisms from various fried food samples were Clostridium perfringens.

\begin{tabular}{ll}
\hline Name of the test & Observed results \\
\hline Growth conditions & Strict anaerobic at $37^{\circ} \mathrm{C}$ \\
Growth in selective TSC agar & Black colour colonies \\
Gram staining & Gram positive, large rods \\
Motility & Non motile \\
Spore staining & Presence of spores \\
Capsule staining & Presence of capsule \\
Blood agar & $\beta$-hemolytic, double zone of haemolysis \\
Iron milk test & Presence of stormy fermentation \\
Nitrate reduction & Reduced nitrate to nitrite \\
Lactose gelatin medium & Lactose fermentation and acid production \\
Gelatin liquefaction & Liquefied gelatin after 48 hrs. \\
Indole & Negative \\
Methyl red & Positive \\
Vogus Proskauer & Negative \\
Hydrogen Sulphide & Positive \\
Carbohydrate Formation & Ferment glucose, sucrose, lactose , maltose produced acid and gas \\
Egg-yolk milk agar & Lecithinase activity is observed \\
Toxin production & Positive \\
\hline
\end{tabular}

Table 2: The binding energies (kcal/mol) of various plant derived compounds with deltatoxin after molecular docking. All molecules satisfy the drug likeness properties evaluated by Lipinski rule of 5 showing zero violation. Curcumin, Eugenol, Palmatine, Eucalyptol, Chrysin etc. have high ability to interact with the target protein than antibiotics (not shown in the table, the binding energies of five antibiotics used in the study are Linezolid-6.08, Clindamycin-5.49, Penicillin5.01 , Chloramphenicol-4.17 and Metronidazole-3.67 kcal/mol). Our study concluded that plant derived compounds have better efficiency to bind the toxin than antibiotics; Curcumin is best plant extract than the antibiotic Linezolid.

\begin{tabular}{|c|c|c|c|c|c|}
\hline $\begin{array}{l}\text { Plant Extracts \& } \\
\text { Extracts }\end{array}$ & Source of the Medicinal Plant & Common Name & $\begin{array}{l}\text { Lipinski } \\
\text { Rule of } 5 \\
\text { (Number of } \\
\text { violation) }\end{array}$ & $\begin{array}{l}\text { Accession Number } \\
\text { of ligand } \\
\text { (Chemspider } \\
\text { Database) }\end{array}$ & $\begin{array}{l}\text { Docking Binding } \\
\text { Energy } \\
(\mathrm{kcal} / \mathrm{mol})\end{array}$ \\
\hline Curcumin & Curcuma longa & Turmeric & 0 & 839564 & 8.59 \\
\hline Eugenol & Commiphora myrrha & Gum myrrh & 0 & 13876103 & 6.18 \\
\hline Palmatine & Phellodendron amurense & Amur cork tree & 0 & 17947 & 5.72 \\
\hline Eucalyptol & Eucalyptus globulus & Eucalyptus & 0 & 21111689 & 5.69 \\
\hline Chrysin & Passiflora coerulea & Blue passion flower & 0 & 4444926 & 5.42 \\
\hline Thujone & $\begin{array}{l}\text { Citrus reticulata var. } \\
\text { madurensis }\end{array}$ & Variety of the mandarin orange & 0 & 229574 & 5.33 \\
\hline Violaxanthin & Cucurbita реро & $\begin{array}{l}\text { Varieties of squash, gourd, and } \\
\text { pumpkin }\end{array}$ & 0 & 395237 & 5.23 \\
\hline Warfarin & Anthoxanthum odoratum & Vanilla grass & 0 & 10442445 & 5.22 \\
\hline Lutein & Cucurbita pepo & $\begin{array}{l}\text { Varieties of squash, gourd, and } \\
\text { pumpkin }\end{array}$ & 0 & 4444655 & 5.20 \\
\hline Borneol & Cymbopogon nardus & Lemon grass & 0 & 5026296 & 5.16 \\
\hline Artemisin & Artemisia annua & Sweet wormwood & 0 & 58542 & 5.14 \\
\hline Bergapten & Citrus aurantium & Seville orange & 0 & 2265 & 5.09 \\
\hline Benzoyl Peroxide & Hibiscus rosasinensis & Hibiscus & 0 & 6919 & 5.07 \\
\hline Pinene & Aniba rosaeodora & Magnoliid & 0 & 6402 & 5.06 \\
\hline Cadinane & Commiphora myrrha & Gum myrhh & 0 & 7827631 & 5.01 \\
\hline Ficusin & Ficus septica & Septic fig & 0 & 5964 & 5.01 \\
\hline Ledol & Eucalyptus polybractea & Eucalyptus & 0 & 91904 & 4.98 \\
\hline Cedrol & Cupressus sempervirens & Italian cypress & 0 & 59018 & 4.92 \\
\hline Phellandrene & Eucalyptus polybractea & Eucalyptus & 0 & 7180 & 4.89 \\
\hline Limonene & $\begin{array}{l}\text { Citrus reticulata var. } \\
\text { madurensis }\end{array}$ & Variety of the Mandarin orange & 0 & 20939 & 4.79 \\
\hline Osthol & Citrus aurantium & Sweet orange & 0 & 9811 & 4.66 \\
\hline Caryophyllene & Cymbopogon martinii & Lemon grass & 0 & 4444848 & 4.57 \\
\hline Harmane & Passiflora coerulea & Blue passion flower & 0 & 4444755 & 4.57 \\
\hline Asarone & Coriandrum sativum & Coriander & 0 & 552532 & 4.49 \\
\hline Neocnidilide & Apium graveolens & Celery & 0 & 2341004 & 4.42 \\
\hline Theophylline & Camellia sinensis & Chinese tea & 0 & 2068 & 4.35 \\
\hline Carvacrol & Origanum vulgare & Oregano & 0 & 21105867 & 4.31 \\
\hline Coumaric acid & Leptospermum polygalifolium & Yellow tea & 0 & 553146 & 4.3 \\
\hline Theobromine & Theobroma cocao & Cocao plant & 0 & 5236 & 4.28 \\
\hline
\end{tabular}

ISSN 0973-2063 (online) 0973-8894 (print) 


\begin{tabular}{|c|c|c|c|c|c|}
\hline Pinocarvone & Eucalyptus polybractea & Eucalyptus & 0 & 108603 & 4.18 \\
\hline $\begin{array}{l}\text { 3,4Dihydroxycinnamic } \\
\text { Acid }\end{array}$ & Melissa officinalis & Lemon balm & 0 & 200866 & 4.12 \\
\hline Senkyunolide & Apium graveolens & Garden celery & 0 & 151725 & 4.11 \\
\hline Carveol & Eucalyptus polybractea & Eucalyptus & 0 & 7160 & 4.05 \\
\hline Cuminaldehyde & Commiphora myrrha & Gum myrrh & 0 & 320 & 4.05 \\
\hline Methylisoeugenol & Daucus carota & Carrot & 0 & 20473735 & 4.05 \\
\hline Terpinenol & Artemisia princeps & Japanese mugwort & 0 & 10756 & 4.05 \\
\hline Linalool & Cupressus sempervirens & Italian cypress & 0 & 13849981 & 4.02 \\
\hline Anisic acid & Pimpinella anisum & Flowering plant & 0 & 10181338 & 4.00 \\
\hline Carvone & Eucalyptus polybractea & Eucalyptus & 0 & 7161 & 4.00 \\
\hline Apiol & Apium graveolens & Garden celery & 0 & 10209 & 3.99 \\
\hline Capsaicin & Capsicum species & Capsicum & 0 & 1265957 & 3.98 \\
\hline Cryptone & Angelica archangelica & Angelica & 0 & 83754 & 3.94 \\
\hline Caffeic Acid & Melissa officinalis & Lemon balm & 0 & 600426 & 3.92 \\
\hline Ocimene & Apium graveolens & Celery & 0 & 4520017 & 3.84 \\
\hline Methyleugenol & Daucus carota & Carrot & 0 & 10605849 & 3.83 \\
\hline Niacin & Vitis vinifera & Grape & 0 & 913 & 3.8 \\
\hline Geranyl Acetate & Cymbopogon nardus & Lemon grass & 0 & 1266019 & 3.77 \\
\hline Verbenene & Eucalyptus polybractea & Eucalyptus & 0 & 10260983 & 3.76 \\
\hline Thymol & Thymus vulgaris & Thyme & 0 & 21105998 & 3.73 \\
\hline Camphene & Angelica archangelica & Angelica & 0 & 6364 & 3.72 \\
\hline Carene & Angelica archangelica & Angelica & 0 & 24263 & 3.72 \\
\hline Estragole & Artemisia dracunculus & Dragon's-wort & 0 & 13850247 & 3.61 \\
\hline Cinnamaldehyde & Cinnamomum species & Cinnamon & 0 & 553117 & 3.57 \\
\hline Neryl Acetate & Citrus aurantifolia & Citrus fruits & 0 & 1266018 & 3.56 \\
\hline Terpinolene & $\begin{array}{l}\text { Citrus reticulata var. } \\
\text { madurensis }\end{array}$ & Variety of the Mandarin orange & 0 & 10979 & 3.498 \\
\hline Cymene & Angelica archangelica & Angelica & 0 & 7183 & 3.47 \\
\hline Catechol & Mimosa catechu & Black cutch & 0 & 13837760 & 3.45 \\
\hline Methyl Benzoate & Antirrhinum majus & Snapdragon & 0 & 6883 & 3.38 \\
\hline Methylanisole & Amorphophallus albispathus & Bagana (grown in Ethiopia) & 0 & 21105959 & 3.31 \\
\hline Sabenene & Apium graveolens & Garden celery & 0 & 17769 & 3.29 \\
\hline Citral & Cymbopogon nardus & Lemon grass & & 558878 & 3.28 \\
\hline Cinnamyl alcohol & Cinnamomum species & Cinnamon & 0 & 21105870 & 3.26 \\
\hline Methylpyrrolidine & Solanaceae families & Flowering plants & 0 & 8143 & 3.232 \\
\hline Cresol & Commiphora myrrha & Gum myrrh & 0 & 13839082 & 3.23 \\
\hline Citronellol & Cymbopogon nardus & Lemon grass & 0 & 13850135 & 3.10 \\
\hline Berberine Sulfate & Berberis vulgaris & Barberry & 0 & 11948 & 2.96 \\
\hline Geraniol & Cymbopogon nardus & Lemon grass & 0 & 13849989 & 2.88 \\
\hline Myrcene & Citrus paradisi & Grape fruit & 0 & 28993 & 2.83 \\
\hline Decenol & Brassica oleracea & Cabbage & 0 & 4517049 & 2.28 \\
\hline Berberine Iodide & Mahonia aquifolium & Oregon-grape & 0 & 65288 & 1.04 \\
\hline
\end{tabular}

